\title{
The Role of Taste in Cephalic Phase of Insulin Secretion
}

\section{Dušková M.1, Macourek M.2, Šrámková M.1, Hill M.1, Stárka L.1}

${ }^{1}$ Institute of Endocrinology, Prague, Czech Republic;

${ }^{2}$ Grammar School Botičská, Prague, Czech Republic

Received June 7, 2013; Accepted November 25, 2013.

Key words: Sweet taste - Insulin - Cortisol - C-peptide

Abstract: The effect of a short gustatory signal of a sweet solution was tested on 15 young male volunteers. The experiment consisted of mouth rinsing with either a sucrose or aspartate solution or pure water as a placebo. Blood was then taken in short intervals of 0, 5, 10,15 and $20 \mathrm{~min}$. Blood glucose, C-peptide, insulin and cortisol were determined. While C-peptide and glucose were unaffected, a short-term increase in insulin was observed after the sucrose, but not after the aspartate or placebo. The increase in insulin was significant, though it amounted to only $0.5 \mathrm{mIU} / \mathrm{l}$ and lasted approx. $15 \mathrm{~min}$ reaching then the starting value. The decline of cortisol level within 20 min of the experiment was approx. $40 \mathrm{nmol} / \mathrm{l}$, although it was also observed after aspartate or placebo mouth rinsing and was probably caused by stress factors or anticipation. In conclusion, the contribution of taste to the cephalic phase of insulin secretion is small yet significant, and mouth rinsing with $5 \%$ sucrose causes an insulin increase of just under $1 \mathrm{IU} / \mathrm{l}$, which returns to starting level within $15 \mathrm{~min}$.

This study was supported by the Ministry of Health, Czech Republic - conceptual development of research organization (Institute of Endocrinology EU IN 00023761), and by grant NT-11277-6 of the Internal Grant Agency of MZ ČR.

Mailing Address: Prof. RNDr. Luboslav Stárka, MD., DSc., Institute of Endocrinology, Národní 8, 11694 Prague 1, Czech Republic; e-mail: Istarka@endo.cz 


\section{Introduction}

Secretion of insulin by beta-cells of the islets of Langerhans is a very complex dynamic process that includes basal and stimulated insulin secretion. Two phases, one early and one late, can be distinguished in insulin secretion initiated by various stimuli. The early phase is characterised by the secretion of preformed insulin granules, lasts about 15 minutes and is formed by cephalic (Berthoud et al., 1981; Ahrén and Holst, 2001; Gautam et al., 2006) and gastrointestinal components.

The cephalic phase of insulin secretion starts by stimulating visual, olfactory and taste receptors (Berthoud et al., 1981; Ahrén and Holst, 2001; D'Alessio et al., 2001) and therefore is not only a part of the first steps of food ingestion, but also of anticipatory physiological regulation in feeding (Power and Schulkin, 2008). Though taste is a very important factor for appetite and food intake, its separate role in the secretion of hormones taking part in glucose homeostasis has not been studied much in detail until now. The quantitative contribution of taste to changes in circulating hormonal levels in humans is, in particular, unknown.

The role of the gustatory system on glucose homeostasis in humans was documented mainly postingestively on intestinal taste receptors (Maillet, 2011; Merigo et al., 2011; Shirazi-Beechey et al., 2011; Geraedts et al., 2012). The study of Joosten et al. (2010) sought to determine whether short oral alcohol exposure, in the form of white wine, provokes cephalic phase responses. The exposure transiently lowered serum free fatty acids. Another study investigated the changes in athletic performance after oral carbohydrate sensing. Simple tasting (mouth rinsing) with saccharides had the same effect on increasing performance as the ingestion of a beverage (Jeukendrup and Chambers, 2010).

The aim of the present study is to show to what extent the concentrations of insulin, C-peptide and cortisol are changed by a simple mouth rinsing with a sucrose or sweetener solution.

\section{Material and Methods}

Subjects

15 non-obese voluntary male participants aged $20-30$ years were included in the study. Their average age was $28.8 \pm 6.32$ years, BMI $23.43 \pm 1.71 \mathrm{~kg} / \mathrm{m}^{2}$. Men were randomly selected in three groups. In the first group, the tests were carried out in the sequence sugar - sweetener - water; in the second in the sequence placebo - sweetener - sugar and, in the third, water - sugar - sweetener. A $5 \%$ sucrose and $0.018 \%$ aspartate solution was used for the sweet solution. Tap water was used as a placebo. The study was improved by the Ethical Committee of the Institute of Endocrinology and all participants signed the informed consent.

The experiment started at 8 a.m.; the overnight-fasting volunteers were laid down and their forearm veins were cannulated and, following $15 \mathrm{~min}$ of rest, the zero-time blood withdrawal was carried out. The first mouth rinsing then started with blood withdrawals at 5, 10,15 and $20 \mathrm{~min}$. A 10-min pause was followed by 
the next rinsing. Serum was separated and glucose, insulin, C-peptide and cortisol were determined in all samples.

\section{Analytical methods}

Blood glucose was measured using the enzymatic reference method with hexokinase (analyzer Cobas Integra 400 plus, Roche). The measuring range of the kit was $0.12-40 \mathrm{mmol} / \mathrm{I}$. Intra- and inter-set reproducibility was $1.7 \%$ and $2.6 \%$, respectively.

C-peptide was measured using ECLIA (electrochemiluminescence immunoassay, analyzer Modular E 170, Roche). The measuring range of the kit (defined by the lower detection limit and the maximum of the master curve) was $0.003-13.3 \mathrm{nmol} / \mathrm{l}$ for plasma. Intra- and inter-assay coefficient of variation was $1.5 \%$ and $2.3 \%$, respectively.

Insulin was measured using ECLIA (electrochemiluminescence immunoassay, analyzer Modular E 170, Roche). The measuring range of the kit (defined by the lower detection limit and the maximum of the master curve) was $0.2-1,000 \mathrm{IU} / \mathrm{ml}$. Cortisol was assayed using a RIA kit from Orion, Finland (intra-assay CV $=3.8 \%$, inter-assay $\mathrm{CV}=4.4 \%)$.

\section{Statistical data analysis}

Repeated measures ANOVA with subject factor and within-subject factor Time was used to evaluate the differences between the experiment stages followed by least significant difference (LSD) multiple comparisons. The original dependent variables and the covariate were transformed by a power transformation to attain a constant variance and symmetric distribution of the data and residuals (Meloun et al., 2000). Statistical software Statgraphic Centurion version XVI (Herndon, VA, USA) was used for the calculations. The homogeneity of the data and residual were checked as described elsewhere (Meloun et al., 2002).

\section{Results}

To prove effect of mouth rinsing with water and sugar or sweetener solutions, the time course analysis by ANOVA was decisive (see: Time in Figures 1-4).

Oral stimulation by rinsing mouth with a $5 \%$ sucrose solution caused a slight but significant rise in insulin level in circulating blood (Figure 1) over the course of 5-10 minutes and then returned to the basal level. No change in glucose and C-peptide levels were observed (Figures 2 and 3). Sucrose rinsing also caused a distinct drop in cortisol level, though this effect was observed also with the sweetener and placebo (Figure 4). This probably indicates an unspecific effect.

Less pronounced changes in insulin concentration caused by the sweetener (aspartate) solution in an organoleptic concentration similar to the sucrose solution were insignificant. Aspartate decreased significantly the time course of C-peptide (Figure 3).

The placebo (water) had no effect on circulating insulin or C-peptide concentrations. 


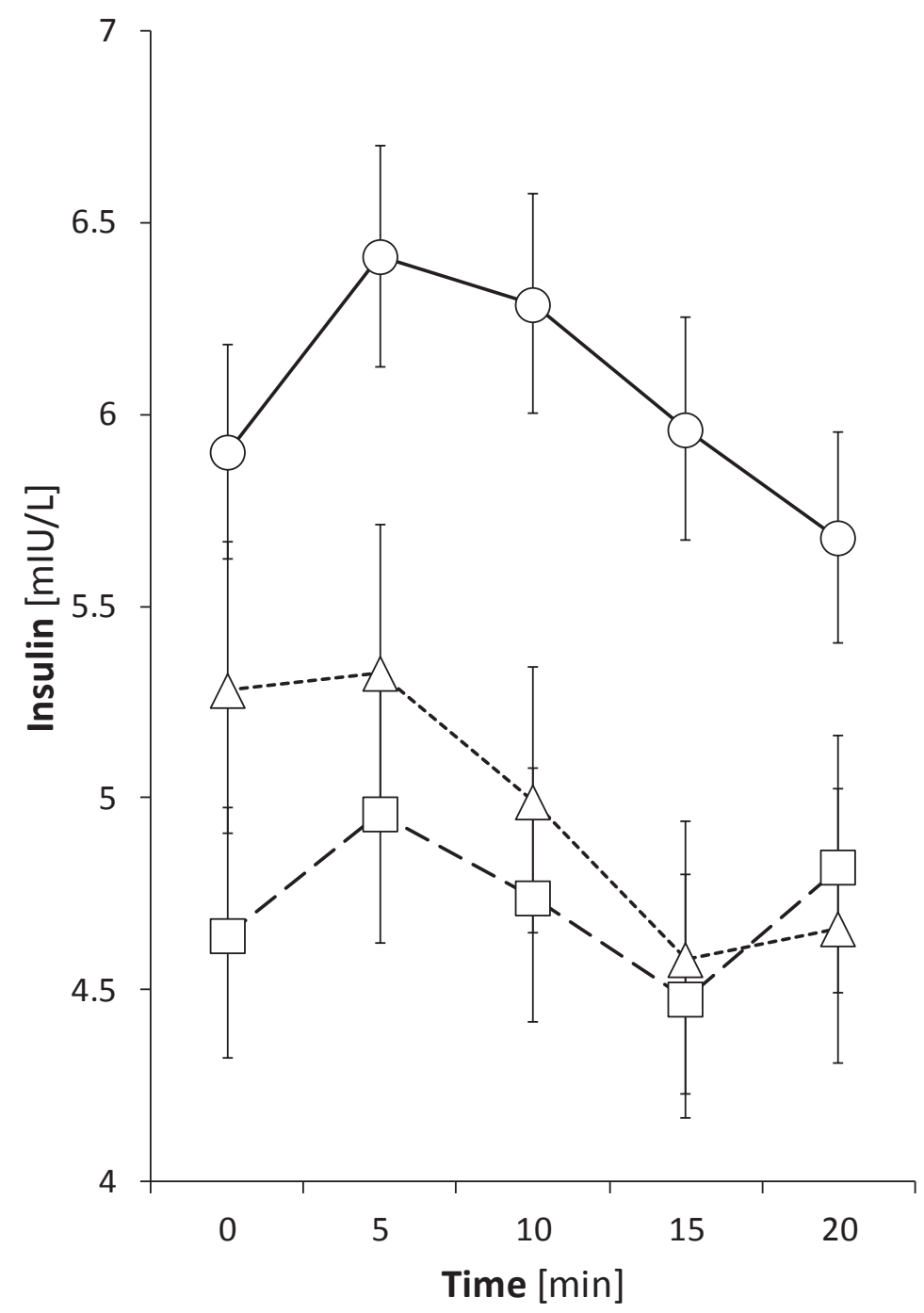

Figure 1 - Changes of insulin after stimulation with sugar, sweetening agent, and without the stimulation evaluated by repeated measures ANOVA consisting of subject factor and a within-subject factor Time and followed by a least significant difference multiple comparisons. Circles, squares, and triangles with error bars represent mean values with their $95 \%$ confidence intervals for sugar, sweetening agent, and no stimulation, respectively. Statistics for individual stimulations are as follows:

Sugar stimulation: $R^{2}=94.5 \%, p<0.0001$; Time: $F=2.26, p=0.0741$; Subj.: $F=66.37, p<0.0001$

Sweetening agent stimulation: $R^{2}=88.2 \%, p<0.0001$; Time: $F=0.61, p=0.6555$; Subj.: $F=29.66, p<0.0001$

Placebo (water): $R^{2}=87.2 \%, p<0.0001$; Time: $F=1.75, p=0.1605$; Subj.: $F=24.01, p<0.0001$

$R^{2}, F$, and $p$ represent the $\%$ of the total variability in dependent variable explained by the ANOVA model, F-ratio, and p-level, respectively.

— full line with circles: sucrose

- - - - dashed line with squares: sweetener

dotted line with triangles: wate

Insulin Secretion and Taste 


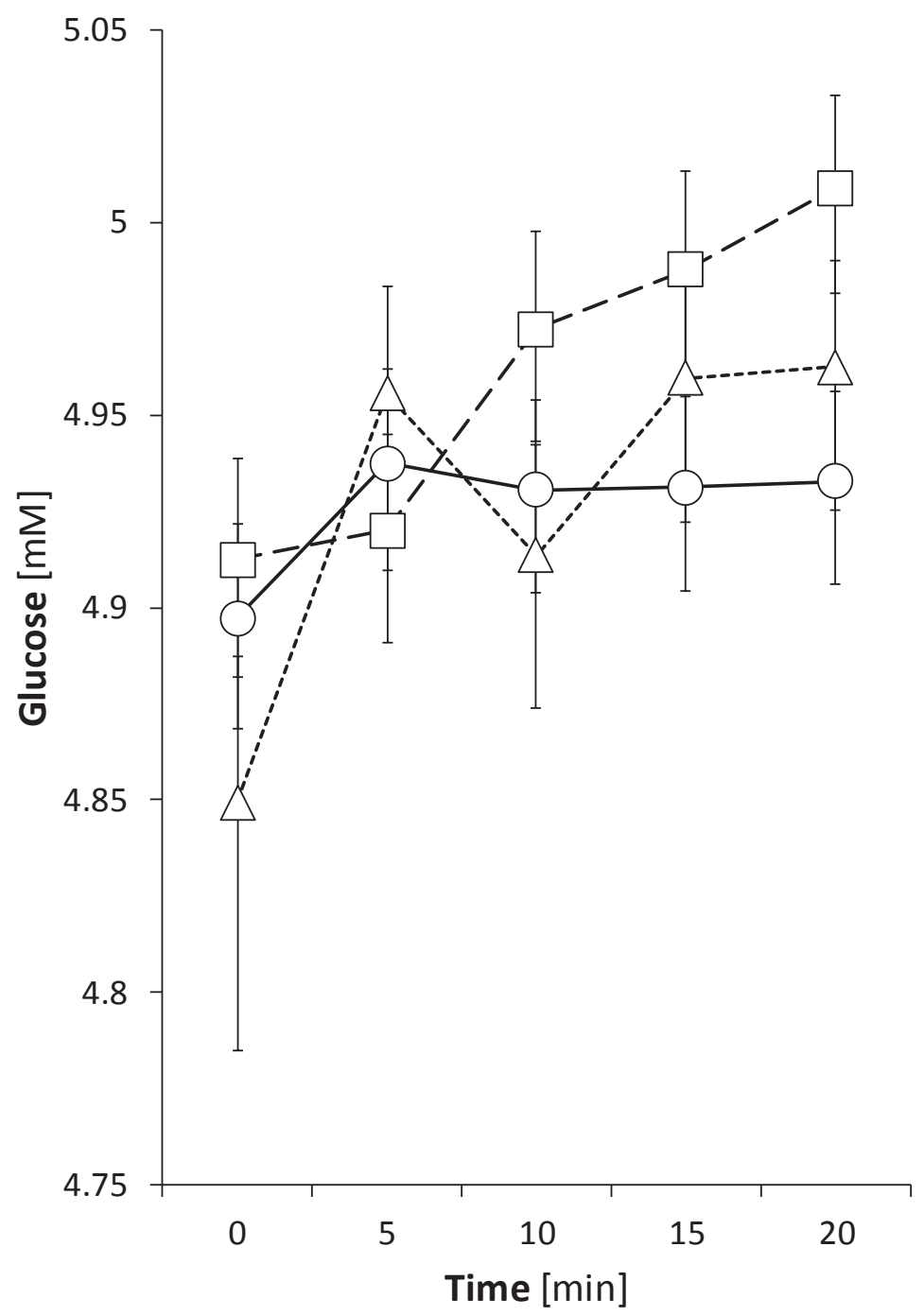

Figure 2 - Changes of glucose after stimulation with sugar, sweetening agent, and without the stimulation evaluated by a repeated measures ANOVA consisting of subject factor and a within-subject factor Time and followed by a least significant difference multiple comparisons. Circles, squares, and triangles with error bars represent mean values with their $95 \%$ confidence intervals for sugar, sweetening agent, and no stimulation, respectively. Statistics for individual stimulations are as follows:

Sugar stimulation: $R^{2}=96.6 \%, p<0.0001$; Time: $F=0.78, p=0.5403$; Subj.: $F=111.6, p<0.0001$

Sweetening agent stimulation: $R^{2}=95.5 \%, p<0.0001$; Time: $F=5.06, p=0.0016$; Subj.: $F=76.92, p<0.0001$

Placebo (water): $R^{2}=96.0 \%, p<0.0001$; Time: $F=3.22, p=0.0233$; Subj.: $F=85.31, p<0.0001$

$R^{2}, F$, and $p$ represent the \% of the total variability in dependent variable explained by the ANOVA model, F-ratio, and p-level, respectively.

_ full line with circles: sucrose

- - - - dashed line with squares: sweetener dotted line with triangles: wate

Dušková M.; Macourek M.; Šrámková M.; Hill M.; Stárka L. 


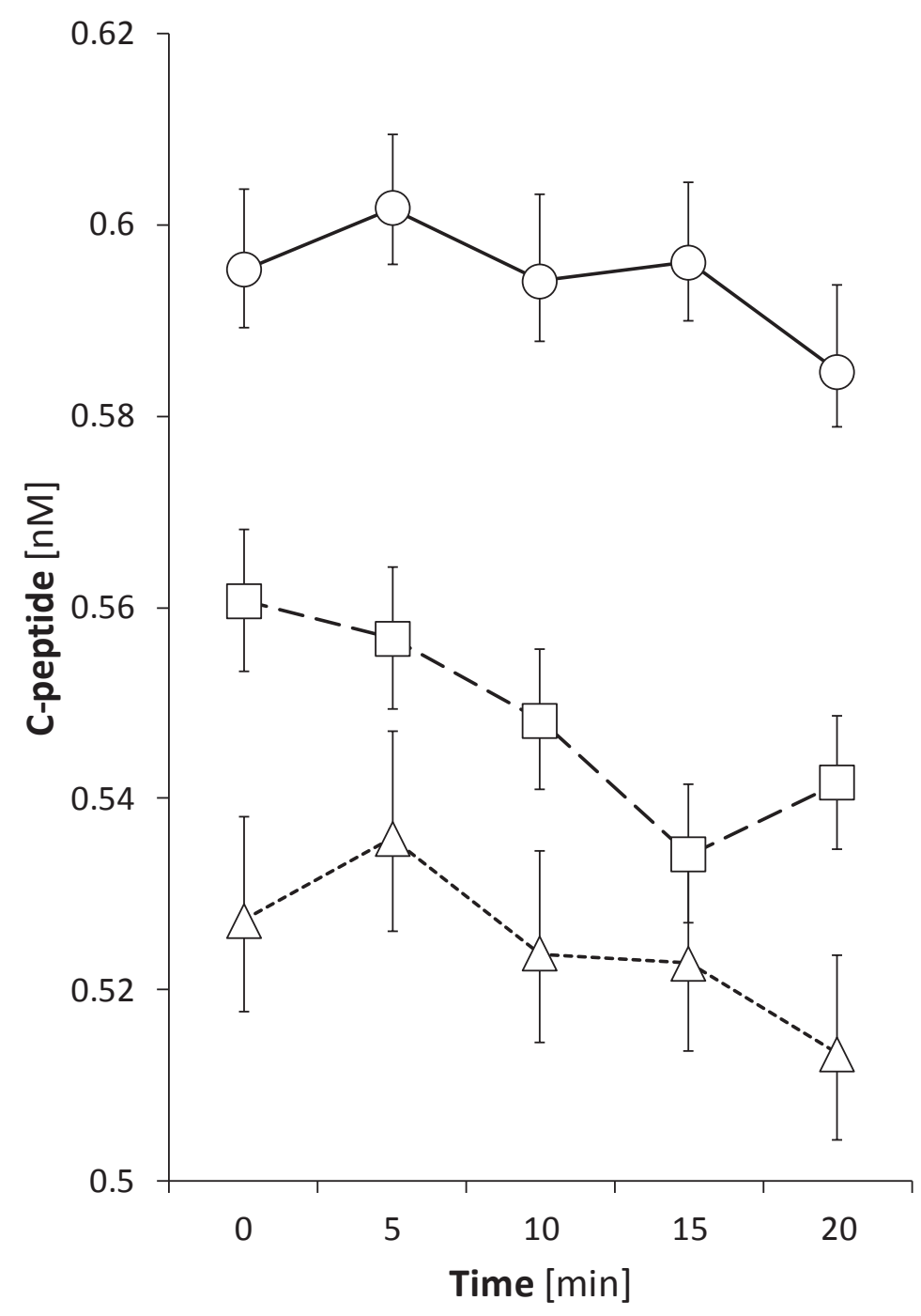

Figure 3 - Changes of C-peptide after stimulation with sugar, sweetening agent, and without the stimulation evaluated by a repeated measures ANOVA consisting of subject factor and a within-subject factor Time and followed by a least significant difference multiple comparisons. Circles, squares, and triangles with error bars represent mean values with their $95 \%$ confidence intervals for sugar, sweetening agent, and no stimulation, respectively. Statistics for individual stimulations are as follows:

Sugar stimulation: $R^{2}=99.2 \%, p<0.0001$; Time: $F=1.87, p=0.1297$; Subj.: $F=464.79, p<0.0001$

Sweetening agent stimulation: $R^{2}=98.6 \%, p<0.0001$; Time: $F=4.36, p=0.0041 ;$ Subj.: $F=272.72, p<0.0001$

Placebo (water): $R^{2}=97.2 \%, p<0.0001$; Time: $F=1.38, p=0.2589$; Subj.: $F=144.37, p<0.0001$

$R^{2}, F$, and $p$ represent the \% of the total variability in dependent variable explained by the ANOVA model, F-ratio, and $p$-level, respectively.

— full line with circles: sucrose

- - - - dashed line with squares: sweetener dotted line with triangles: wate

Insulin Secretion and Taste 


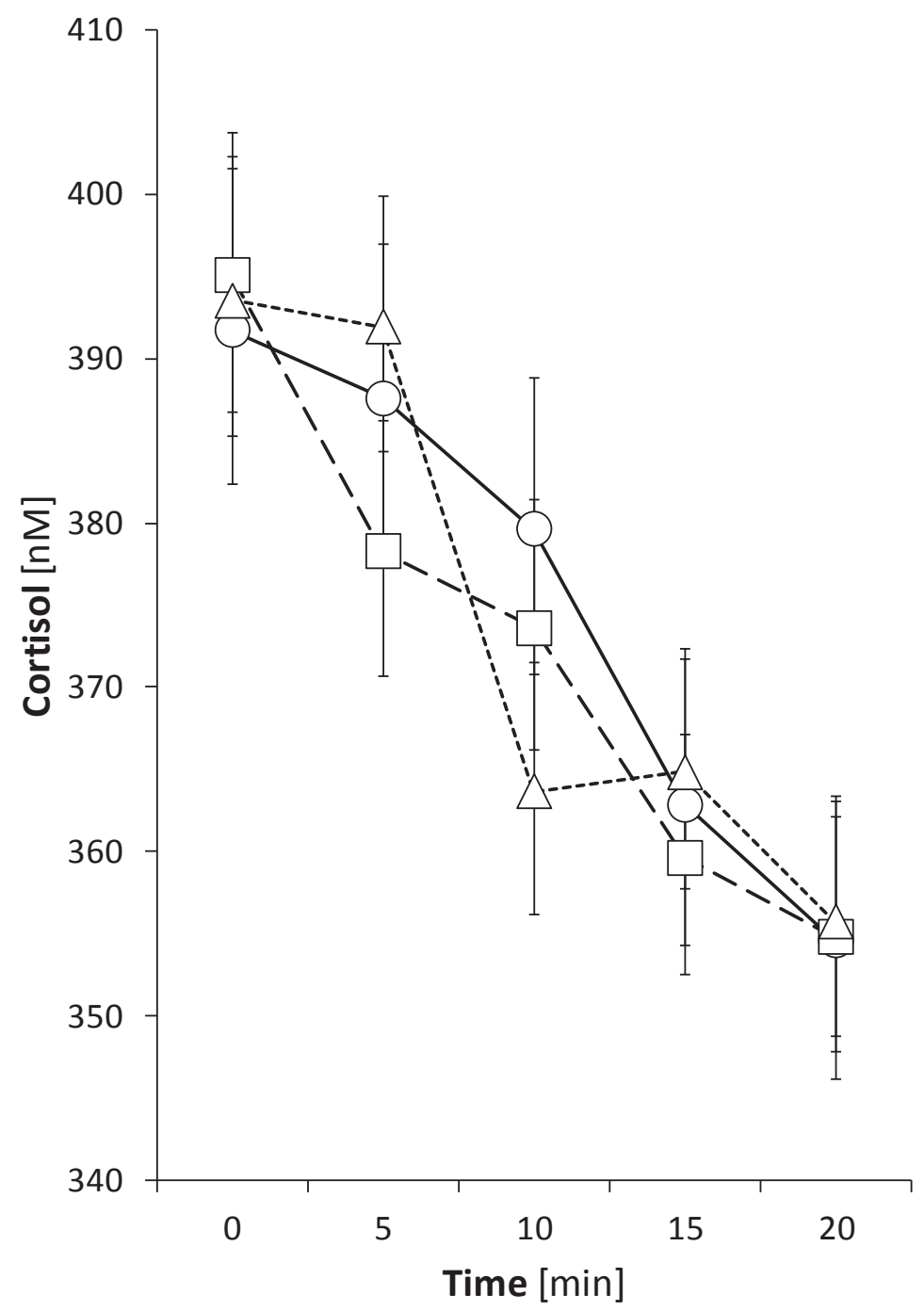

Figure 4 - Changes of cortisol after stimulation with sugar, sweetening agent, and without the stimulation evaluated by a repeated measures ANOVA consisting of subject factor and a within-subject factor Time and followed by a least significant difference multiple comparisons. Circles, squares, and triangles with error bars represent mean values with their $95 \%$ confidence intervals for sugar, sweetening agent, and no stimulation, respectively. Statistics for individual stimulations are as follows:

Sugar stimulation: $R^{2}=97.4 \%, p<0.0001$; Time: $F=6.37, p=0.0003$; Subj.: $F=150.35, p<0.0001$

Sweetening agent stimulation: $R^{2}=97.8 \%, p<0.0001$; Time: $F=8.44, p<0.0001$; Subj.: $F=172.85, p<0.0001$

Placebo (water): $R^{2}=98.6 \%, p<0.0001$; Time: $F=8.39, p=0.0001$; Subj.: $F=234.78, p<0.0001$

$R^{2}, F$, and $p$ represent the \% of the total variability in dependent variable explained by the ANOVA model, F-ratio, and p-level, respectively.

\footnotetext{
_ full line with circles: sucrose

dashed line with squares: sweetener

dotted line with triangles: wate
}

Dušková M.; Macourek M.; Šrámková M.; Hill M.; Stárka L. 


\section{Discussion}

Attention was recently paid to glucose sensing and signalling in the post-ingestive phase and to the regulation of intestinal glucose transport (Maillet, 2011; ShiraziBeechey et al., 2011; Geraedts et al., 2012). In this respect the lingual tasting system warranted less attention, though the presence of various hormones (Martin et al., 2009) and glucose transporters (Merigo, 2011) was demonstrated in taste receptor cells. The presence of glucagon-like peptide GLP-1 in taste cells type II and III highlights in particular a parallel between gustatory and intestinal epithelia (Martin et al., 2009).

The cephalic phase of insulin secretion is commonly ascribed to the complex signals from the visual, gustatory and olfactory system together with anticipation based on previous experience. In the present study, we try to show that the signalling by isolated gustatory perception of sweet taste contributes significantly, though only in a small extent and briefly. Such effect lasts only minutes, similarly as in the experiments with short oral white wine exposure (Joosten et al., 2010), which provoked transient decrease of serum free fatty acids. It should be mentioned that liquids and chewing gum do not provide adequate stimulation for vagal efferent activation of nutrient metabolism in humans and that mixed nutrient foods are the optimal stimuli (Teff, 2010). The effect of insulin increase induced by taste sensing as a part of cephalic phase is far less intensive than stimulation of insulin secretion by gastrointestinal regulatory factors in the later acute phase of insulin secretion. There is a discrepancy between increase of insulin after sweet sensing and permanent levels of C-peptide, suggesting a mobilisation of insulin from preformed granules.

The present study showed nearly constant glucose and C-peptide level during the experiment, a short insulin increase after sucrose signal and a decrease of the cortisol concentration, which, however, was also observed after placebo exposure. We tried to eliminate the effect of cortisol daily rhythm by changing the sequence of application of the three signals (sugar - sweetener - water); some effect could be expected as consequence of cannulation (Šimůnková et al., 2010), but, despite this, we are not able to explain the cortisol decrease fully.

Our results are in agreement with the experience that in comparison with sucrose, sweeteners are metabolically less active (Pepino and Bourne, 2011).

In conclusion, the contribution of taste to the cephalic phase of insulin secretion is small yet significant, and mouth rinsing with $5 \%$ sucrose causes an insulin increase of just under $1 \mathrm{mlU} / \mathrm{l}$, which returns to starting level within $15 \mathrm{~min}$.

\section{References}

Ahrén, B., Holst, J.J. (2001) The cephalic insulin response to meal ingestion in humans is dependent on both cholinergic and noncholinergic mechanisms and is important for postprandial glycemia. Diabetes 50(5), 1030-1038.

Berthoud, H. R., Bereiter, D. A., Trimble, E. R., Siegel, E. G., Jeanrenaud, B. (1981) Cephalic phase, reflex insulin secretion. Neuroanatomical and physiological characterization. Diabetologia 20, 393-401. 
D’Alessio, D. A., Kieffer, T. J., Taborsky, G. J. Jr., Havel, P. J. (2001) Activation of the parasympathetic nervous system is necessary for normal meal-induced insulin secretion in rhesus macaques. J. Clin. Endocrinol. Metab. 86(3), 1253-1259.

Gautam, D., Han, S. J., Hamdan, F. F., Jeon, J., Li, B., Li, J. H., Cui, Y., Mears, D., Lu, H., Deng, C., Heard, T., Wess, J. (2006) A critical role for beta cell M3 muscarinic acetylcholine receptors in regulating insulin release and blood glucose homeostasis in vivo. Cell Metab. 3(6), 449-461.

Geraedts, M. C., Takahashi, T., Vigues, S., Markwardt, M. L., Nkobena, A., Cockerham, R. E., Hajnal, A., Dotson, C. D., Rizzo, M. A., Munger, S. D. (2012) Transformation of postingestive glucose responses after deletion of sweet taste receptor subunits or gastric bypass surgery. Am. J. Physiol. Endocrinol. Metab. 303(4), E464-E474.

Jeukendrup, A. E., Chambers, E. S. (2010) Oral carbohydrate sensing and exercise performance. Curr. Opin. Clin. Nutr. Metab. Care 13(4), 447-451.

Joosten, M. M., de Graaf, C., Rietman, A., Witkamp, R. F., Hendriks, H. F. (2010) Short-term oral exposure to white wine transiently lowers serum free fatty acids. Appetite 55(1), 124-129.

Maillet, E. L. (2011) Modulation of T1R chemosensory receptors for sweet nutrients - new paradigms in metabolic regulation. Med. Sci. (Paris) 27(2), 177-182. (in French)

Martin, B., Maudsley, S., White, C. M., Egan, J. M. (2009) Hormones in the naso-oropharynx: endocrine modulation of taste and smell. Trends Endocrinol. Metab. 20(4), 163-170.

Meloun, M., Hill, M., Militky, J., Kupka, K. (2000) Transformation in the PC-aided biochemical data analysis. Clin. Chem. Lab. Med. 38, 553-559.

Meloun, M., Militky, J., Hill, M., Brereton, R. G. (2002) Crucial problems in regression modelling and their solutions. Analyst 127, 433-450.

Merigo, F., Benati, D., Cristofoletti, M., Osculati, F., Sbarbati, A. (2011) Glucose transporters are expressed in taste receptor cells. J. Anat. 219(2), 243-252.

Pepino, M. Y., Bourne, C. (2011) Non-nutritive sweeteners, energy balance, and glucose homeostasis. Curr. Opin. Clin. Nutr. Metab. Care 14(4), 391-395.

Power, M. L., Schulkin, J. (2008) Anticipatory physiological regulation in feeding biology: cephalic phase responses. Appetite 50(2-3), 194-206.

Shirazi-Beechey, S. P., Moran, A. W., Batchelor, D. J., Daly, K., Al-Rammahi, M. (2011) Glucose sensing and signalling; regulation of intestinal glucose transport. Proc. Nutr. Soc. 70(2), 185-193.

Šimůnková, K., Hampl, R., Hill, M., Křǐž, L., Hrdá, P., Janíčková-Žd’árská, D., Zamrazil, V., Vrbíková, J., Vondra, K. (2010) Adrenocortical function in young adults with diabetes mellitus type 1.J. Steroid Biochem. Mol. Biol. 22(1-3), 35-41.

Teff, K. L. (2010) Cephalic phase pancreatic polypeptide responses to liquid and solid stimuli in humans. Physiol. Behav. 99(3), 317-323. 